\title{
Scattering of Electromagnetic Wave by system of core/shell microsphere and nanoparticle
}

\author{
Arnold Abramov ${ }^{1}$, Alexander Kostikov², Yutao Yue ${ }^{1}$ \\ ${ }^{1}$ Deep Perception Institute, Wuxi, China \\ ${ }^{2}$ Donbass State Engineering Academy, Kramatorsk, Ukraine \\ *corresponding author, E-mail: ytyue@ustc.edu
}

\begin{abstract}
The paper deals with the scattering of an electromagnetic plane wave by a system of core/shell microsphere and a single nanoparticle placed outside the spheres. Backscattering intensity perturbation caused by the nanoparticle was studied, and it was found that the position of the inner sphere can be used as a new effective tool to both detect a particle and to determine its size. The system of core/shell microsphere provides a longer particle detection distance compared with a single sphere system. It is shown that the maximum value of the perturbation of backscattering is reached at the interspherical distance corresponding to the maximal energy of the electromagnetic wave concentrated in the vicinity of the nanojet.
\end{abstract}

\section{Introduction}

The scattering of electromagnetic wave has been studied for more than 100 years. However, it was only in this century that the so called nanojet, a narrow, high-intensity beam of light generated at the shadow-side surface of plane-waveilluminated dielectric cylinders, was discovered [1]. Research has shown that photonic nanojet can be formed by spheres [2], multilayers cylinders [3], discs [4], and dielectric cuboids [5]. The effects of various factors on nanojet parameters have also been studied [6-11]. Considerably interest in nanojet research has been generated because of the possibility for their practical application as follows. Photonic nanojets provide a factor of 26 increases in the volume-integrated electric field within the subwavelength active volume of the photodiode [12]. The potential for portable nanojets by imaging different subwavelength structures was demonstrated in [13]. The effect of photonic nanojets on microsphere imaging has been studied in [14]. Some potential applications were reviewed in [15]. In this work we concentrated on the possibility of using nanojets for the detection of particles that was proposed for the first time in [1]. The authors found that a particle size of $20 \mathrm{~nm}$ can cause a significant (40\%) change in the intensity of the backscattering of an electromagnetic wave by a sphere with a diameter of 2 microns. Currently the use of nanojets for the detection of particles has been confirmed and developed in the work of different authors [16-18].
One can assume that as the greater the intensity and (or) the length of the nanojet, as the greater the backscattering intensity signal should be expected. As it was shown in [19] a longer nanojet can be achieved using two concentric spheres. A more general case has been studied in the [20], in which the authors used a core/shell microsphere system to generate a nanojet, assuming the possibility of changing the position of the inner sphere along the nanojet axis. It has been found that for certain asymmetric positions of the inner sphere ultra long nanojet of 27 wavelengths can be achieved. Further, the core/shell microsphere system has the following advantages for detection compared with a single sphere system: a more powerful and longer nanojet; and the appearance of additional factors affecting the detection position, size and dielectric permeability of the inner sphere. In the present paper we study how a core/shell microsphere system affects the possibility of nanoparticle detection.

\section{Modeling}

Our model consists of sphere $A$, into which sphere $B$ is embedded and small sphere $N$ placed outside of spheres $A$ and $B$ (Fig. 1). Radiuses (in $\mu \mathrm{m}$ ) and refraction indexes for spheres $A$ and $B$ are: $R_{A}=2.65, R_{B}=R_{A} / 2, n_{A}=1.844, n_{B}=1.6$. If the refractive index of a sphere $A$ is less than that of the inner sphere $B$, then this means that the electromagnetic wave propagates from a less dense medium to a denser one. Then the inner sphere scatters incident wave, inhibiting the propagation of the wave through the sphere $A$. Therefore quantities for $n_{A}$ and $n_{B}$ chosen such a way that $n_{A}<n_{B}$, and that values corresponds to those used in $[19,20])$. The gold

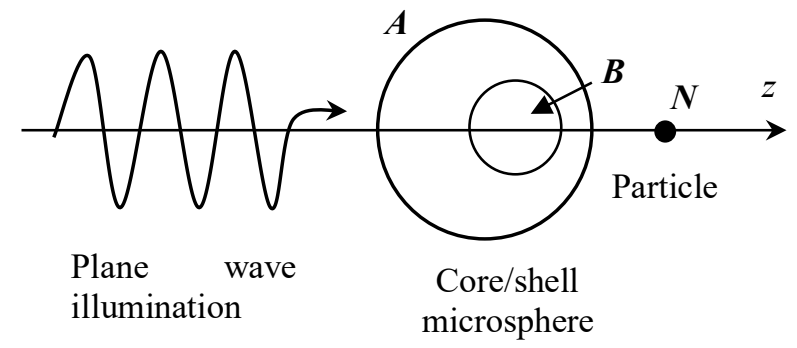

Figure 1: Core $(B) /$ shell $(A)$ microshere and nanoparticle $(N)$ illuminated by plane wave. 
nanoparticle are assumed as sphere $N$ with refractive index $n_{A u}=1.47-i 1.95[1]$. Its size varied in the range of 20-100 $\mathrm{nm}$. A TE-polarized plane wave $(\lambda=532 \mathrm{~nm})$ falls on the group of the spheres positioned on the same axis. The direction of wave propagation is along the axis of symmetry of the spheres and selected as the $z$-axis. The origin of the coordinates corresponds to the centre of sphere $A$.

Following to Ref. [21] Maxwell equations for time harmonic field appear as

$$
\nabla \times\left(\begin{array}{l}
\vec{E} \\
\vec{H}
\end{array}\right)=\frac{k}{1-\beta^{2} m^{2}}\left(\begin{array}{ll}
\beta m^{2} & i \\
-i m^{2} & \beta m^{2}
\end{array}\right) \cdot\left(\begin{array}{l}
\vec{E} \\
\vec{H}
\end{array}\right)
$$

where $m$ is the bulk refractive index of the medium and $\beta$ is dimensionless phenomenological factor. A linear transformation of the electric and magnetic fields of the form

$$
\left(\begin{array}{c}
\vec{E} \\
(\mathrm{i} / \mathrm{m}) \vec{H}
\end{array}\right)=\left(\begin{array}{cc}
1 & 1 \\
1 & -1
\end{array}\right) \cdot\left(\begin{array}{l}
\vec{Q}_{L} \\
\vec{Q}_{R}
\end{array}\right)
$$

will diagonalize Eq.(1), so that

$$
\left.\begin{array}{l}
\nabla \times \vec{Q}_{L}=\mathrm{k}_{L} \cdot \vec{Q}_{L} \\
\nabla \times \vec{Q}_{R}=-\mathrm{k}_{R} \cdot \vec{Q}_{R}
\end{array}\right\}
$$

where the left and right wavenumbers are given by $k_{L}=m k /(1-\beta m), k_{R}=m k /(1+\beta m)$. The functions for $\vec{Q}_{L}$ and $\vec{Q}_{R}$ was sought in the form of an expansion in the vector spherical functions, while expansion coefficients define scattering matrix [21]. The latter allow us to calculate scattering amplitude, i.e. scattering intensity, for any angle. At that scattering intensity for angle $180^{\circ}$ is called backscattering intensity.

According to [1] we choose a normalized backscattering intensity perturbation (BIP) $\Delta I$ as the main parameter characterizing the presence and size of nanoparticles in the vicinity of the photonic nanojet

$$
\Delta I=(I 3-I 2) / I 2,
$$

where $I_{2}$ and $I_{3}$ are the backscattering intensities of the two $(A B)$ and three $(A B N)$ spheres respectively. The quantities $I_{2}$ and $I_{3}$ were calculated independently using Fortran- 90 code for calculation of the electromagnetic scattering developed by D.W. Mackowski and coauthors [22].

\section{Efficiency of core/shell microsphere for nanoparticle detection}

The detailed dependence of the BIP on the intersphere distance $L_{s}$ (between centres of spheres $A$ and $B$ ) for different particle sizes is shown in Fig. 2. Here, for each fixed distance $L_{s}$ we were looking for the maximum value

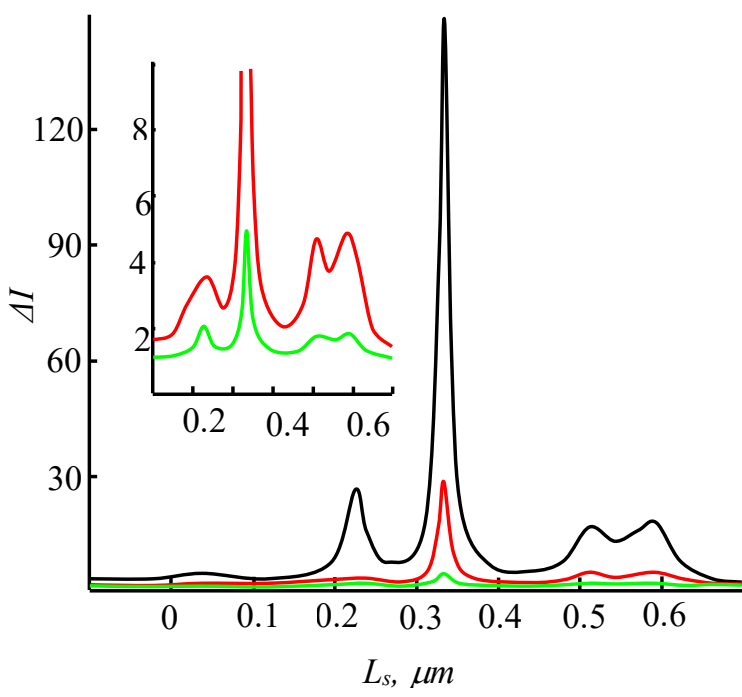

Figure 2: Backscattering intensity perturbation for $A B N$ system versus positions of inner sphere; sizes of gold particle in $\mathrm{nm}: 40$ (green), 60 (red) and 80 (black).

of the BIP varying particle position at a distance of about three wavelengths from the surface of sphere $A$ (area of the nanojet), along $z$ axis as shown on Fig.1. As was expected, the BIP value increases as the size of the nanoparticle increases. However, all other details of dependencies remain the same. It should also be noted that the change in the BIP value occurs at a small interval of the order of wavelength.

In the results presented in this section a particle size of a $60 \mathrm{~nm}$ is chosen as a representative due to a next reason. A BIP value increases significantly as the size of the particle

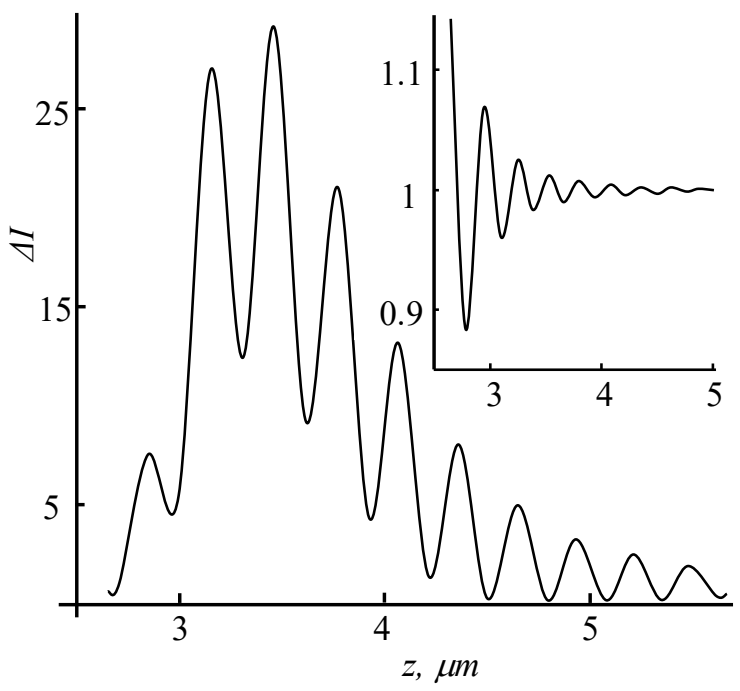

Figure 3: Backscattering intensity perturbation caused by the $60 \mathrm{~nm}$ gold particle and core/shell microsphere $A B . \mathrm{z}$ is the distance between the surface of sphere $A$ and center of the particle. Inset: same for single sphere $A$. 
increases. For instance, as reported in [1] 40\% BIP variation has been calculated for a particle size of $20 \mathrm{~nm}$ while for 100 $\mathrm{nm}$ it increases by orders of magnitude.

Therefore, $60 \mathrm{~nm}$ seems to be the most optimal size for our analysis. This size is also typical for other works that study particle detection. The dependences of the BIP on the particle position for $A N$ and $A B N$ systems are presented in Fig. 3. The position of the inner sphere $B$ is $0.33 \mu \mathrm{m}$ away from the center of sphere $A$ and corresponds to the maximum BIP value in Fig. 2.

In addition to the major difference in the BIP value, other important advantages of the $A B N$ system can be seen: the maximum of BIP values is at a distance of two wavelengths from the edge of the sphere and the BIP is suitable to detect a particle at a distance of up to three wavelengths, while in the case of one sphere $A$ it is fastattenuating from the edge of the sphere.

As it has been shown in [20], the maximum field intensity in the nanojet region increases as the inner sphere moves from left to right. So, placing nanoparticle beside core/shell microsphere we would expect appropriate increase of BIP. However, as can be seen from Fig. 2, the same uniform increase of BIP does not occur. It goes through a maximum value. We found that the value of BIP is determined not by the maximum field intensity in the vicinity of the nanojet, but by the energy of electromagnetic

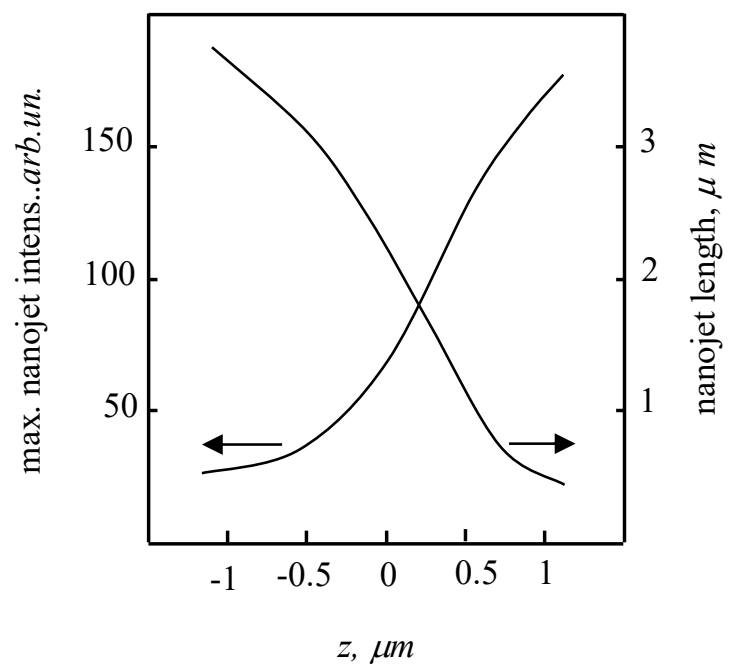

Figure 4: Maximal intensity in the vicinity of nanojet and nanojet length (in the absence of particle) versus positions of the inner sphere $B$.

field accumulated in the nanojet region. In Fig. 4 the maximal intensity in the vicinity of nanojet along with length of nanojet (in the absence of nanoparticle) versus intersphere shifts is shown. It is seen that as the internal sphere shifts, both dependences have regular trend. Thus nor length of nanojet or field intensity in the nanojet area have correlation with BIP. Thus, none of each of that quantity can be responsible for the presence of maximum on Fig. 2. But because of multidirectional trends we can assume that the intensity of the reflected waves will be proportional to the amount of energy stored in the nanojet area. Therefore, we calculated the energy of the electromagnetic wave concentrated in the region of the nanojet. This area was determined by the volume of space where the intensity

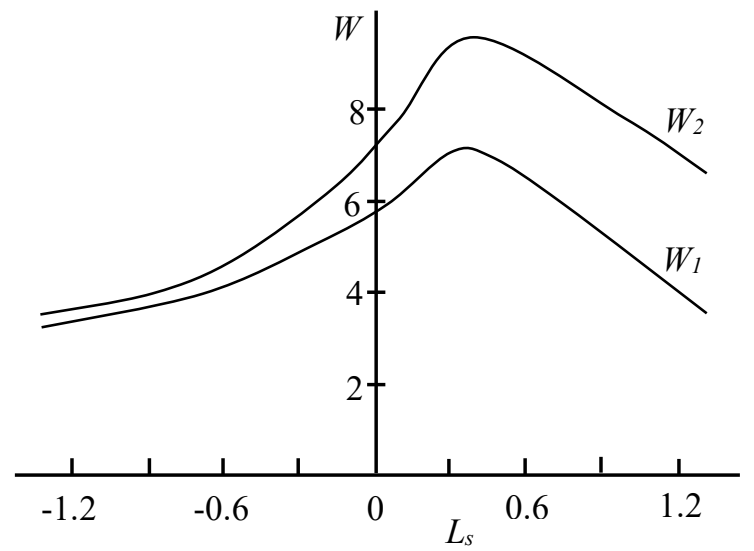

Figure 5: Energy of the electromagnetic wave concentrated in the volume of space where the intensity decreases up to 2 times $\left(W_{l}\right)$ or to value 2 $\left(W_{2}\right)$.

decreases no more than twice $\left(W_{l}\right)$ or to the value $2\left(W_{2}\right)$. The dependence of the values $W_{1}$ and $W_{2}$ on the intersphere distance $L_{c}$ is shown in Fig. 5. Both dependences have a clear maximum just at the value of the intersphere distance for which the BIP maximum was found before $\left(L_{m}\right.$ $\approx 0.33 \mu \mathrm{m})$. This convergence of maximum positions for fundamentally different systems of spheres allows us to make an explicit conclusion about the determining role of energy density in the nanojet area for the maximum BIP value.

Another confirmation of this conclusion is that the maximum position of BIP is the same for different particle sizes (Fig. 2). The changing of full width at half maximum of the dependences is a consequence of different particle sizes. The absence of smoothness in the BIP change is the result of interference effects of scattering of wave groups reflected from the spheres $A$, and $B$ on the one hand and $N$ on the other.

\section{Conclusions}

In this paper, we considered the possibility of detecting a nanoparticle located in the vicinity of a core/shell microsphere system. We found that if an electromagnetic wave is scattered by such a system, the normalized backscattering intensity perturbation has a sharp peak for some intersphere distance. The value of BIP is sufficient to detect a particle at a distance of up to two wavelengths from the surface of the outer sphere. Additional calculations have shown that the maximum value of the BIP is reached at the same interspherical distance at which the energy of the electromagnetic wave in the vicinity of the nanojet is maximal. 


\section{Acknowledgements}

Authors are grateful to Wuxi city government and direction of Jiangsu Industrial Technology Research Institute for appropriate conditions and support to realize this work.

\section{References}

[1] A.-B. Zhang, Z. Chen, A. Taflove, and V. Backman, Photonic nanojet enhancement of backscattering of light by nanoparticles: a potential novel visible-light ultramicroscopy technique, Opt. Express 12(7): 121412202004.

[2] X. Li, Z. Chen, A. Taflove, and V. Backman, Optical analysis of nanoparticles via enhanced backscattering facilitated by 3-D photonic nanojets, Optics Express 13: 526-533, 2005.

[3] V. V. Kotlyar and M. A. Lichmanov, Analysis of electromagnetic wave diffraction on an infinite circular cylinder with several layers of homogeneity, Computer Optics 24: 26-32, 2002.

[4] D. McCloskey, J. J. Wang, and J. F. Donegan, Low divergence photonic nanojets from Si3N4 microdisks, Optics Express 20: 128-140, 2012.

[5] I. V. Minin, O. V. Minin, V. Pacheco-Peca,and M. Beruete, Localized photonic jets from flat, threedimensional dielectric cuboids in the reflection mode, Optics Lett. 40: 2329-2332, 2015.

[6] Y. Liu, B. Wang, and Z. Ding, Influence of incident light polarization on photonic nanojet, Chinese Optics Letters 9: 0729012011.

[7] S.-C. Kong, A. Taflove, and V. Backman, Quasi onedimensional light beam generated by a graded-index microsphere, Optics Express 17: 722-3731, 2009.

[8] C.-Y. Liu, Photonic nanojet enhancement of dielectric microcylinders with metallic coating, $J$. of Optoelectr. and Adv. Mater. 15: 150-154, 2013.

[9] S. Stafeev and V. Kotlyar, Elongated Photonic Nanojet from Truncated Cylindrical Zone Plate, Journal of Atomic, Molecular, and Optical Physics 2012: 1-3, 2012.

[10] J. Schaefer, S.-C. Lee, A. Kienle, Calculation of the near fields for the scattering of electromagnetic waves by multiple infinite cylinders at perpendicular incidence, Journal of Quantitative Spectroscopy \& Radiative Transfer 113: 2113-2123, 2012.

[11] A. Abramov, C. Ji, R. Liu, A. Kostikov, Enhancement of the electromagnetic waves intensity by scattering on multiple infinite cylinders, J. Photonic Mater. Technol. 2: 1-5, 2016.

[12]M. Hasan and J. Simpson, Photonic nanojet-enhanced nanometer-scale germanium photodiode, Applied Optics 52 (22): $5420-5425,2013$.

[13]M. Duocastella, F. Tantussi, A. Haddadpour, R. Zaccaria, A. Jacassi, G. Veronis, A. Diaspro, F. De Angelis, "Combination of scanning probe technology with photonic nanojets," Scientific Reports 7: 1-7, 2017.
[14] S. Yang, F. Wang, Y. - H. Ye, Y.Xia, Y. Deng, J. Wang, Y. Cao, Influence of the photonic nanojet of microspheres on microsphere imaging, Optics Express 25: $27551-27558,2017$.

[15] A. Heifetz, S.-C. Kong, A. V. Sahakian, A. Taflove, and V. Backman, Photonic Nanojets, Journal of Computational and Theoretical Nanoscience 6: 19791992, 2009.

[16]H. Yang, M. Cornaglia, and M. Gijs, Photonic Nanojet Array for Fast Detection of Single Nanoparticles in a Flow, Nano Lett. 15: 1730, 2015.

[17] G. Gu, J. Song, M. Chen,X. Peng, H. Liang, J. Qu, Single nanoparticle detection using a photonic nanojet, Nanoscale 10: 14182 -14189, 2018.

[18] Y. Li, H. Xin, H. Lei, L. Liu, Y. Li, Y. Zhang, B. Li, Manipulation and detection of single nanoparticles and biomolecules by a photonic nanojet, Light: Science \& Applications 5: e16176, 2016.

[19] Y. C. Shen, L. V. Wang, and J. T. Shen, Ultra long photonic nanojet formed by a two-layer dielectric microsphere, Optics Lett. 39: 4120 - 4123, 2014.

[20]P. K. Kushwaha, H. S. Patel, M. K. Swami and P. K. Gupta, Controlled shaping of photonic nanojets using core shell microspheres, Proc. of SPIE 9654: 96541H, 2015.

[21]C. F. Bohren, D. R. Huffman, Absorption and Scattering of Light by Small Particles, Wiley, 1983.

[22]D.W. Mackowski, M.I. Mishchenko, A multiple sphere T-matrix Fortran code for use on parallel computer clusters, Journal of Quantitative Spectroscopy \& Radiative Transfer 112: 2182-2192, 2011. http://www.eng.auburn.edu/ dmckwski/scatcodes/mstm -manual-2013-v3.0.pdf 